\title{
EL PAPEL DE LA MUJER SEMITA EN LA SOCIEDAD MESOPOTÁMICA
}

\author{
THE ROLE OF THE SEMITE WOMAN IN MESOPOTAMIC SOCIETY
}

Efrem Yildiz

Universidad de Salamanca

\section{RESUMEN:}

Este trabajo expone la situación de la mujer semita desdela antigüedad hasta la actualidad, así como un análisis de las leyes y normas que legislaban su situación y posición social a lo largo de la historia. Se analiza también la visión que se le ha dado en la literatura a las mujeres que no vivieron al amparo del hombre, las que pudieron intervenir en la historia. Se crean así las figuras de mujeres guerreras, mujeres heroínas, mujeres gobernantas, aunque estas funciones son excepciones y hechos aislados. Se ahonda también en papel de las religiones en las sociedades semitas actuales y cómo estas influyen en la situación de las mujeres.

\section{Palabras clave:}

mujer semita, mujer en la literatura, religión y sociedad

\section{Abstract:}

This work exposes the situation of the Semitic woman from ancient times to the present, as well as an analysis of the laws and norms that regulated their situation and social position throughout history. The vision that has been given in literature to women who did not live under the protection of men, those who could intervene in history, is also analyzed. Thus the figures of female warriors, female heroines, women rulers are created, although these functions are exceptions and isolated events. It also delves into the role of religions in current Semitic societies and how they influence the situation of women.

\section{KeYWORDS:}

semitic woman, woman in literature, religion and society 
En la evolución de la sociedad semita se puede observar un modus vivendi marcado por la supervivencia y la lucha continua para conservar y proteger al grupo. Este hecho obligó a que las funciones de sus miembros, fueran bien determinadas y de esta forma conservar la pervivencia del colectivo. Una vez asegurada la supervivencia del grupo inicialmente reducido, aparecieron otros factores de tipo económico y territorial que obligaron a multitud de reducidos clanes a unificarse para hacer frente a grupos más poderosos. Por tanto, la fusión de varias agrupaciones minoritarias condujo a la creación de una sociedad que no se limita solamente a la vida nómada o seminómada, sino que también fue diversificándose según las profesiones que desempeñaban sus miembros; de esta forma fueron forjando un sistema social mucho más complejo y amplio que terminó siendo denominado, por los etnógrafos: la confederación.

En esta primera fase, la atención dedicada a la mujer se centra en primer lugar en la protección no solamente física, sino también de su integridad. En otras palabras, se presenta una imagen de un ser humano necesitado de una protección especial por la función que desempeña en la sociedad semita antigua.

Conforme a esta visión, la imagen de la mujer semita se centra en determinados aspectos de la vida, entre los cuales se subraya la transmisión de la vida y por tanto la continuidad. Esta continuidad y posterior ampliación numérica del grupo y sus intereses, hizo que predominaran ciertos aspectos en esta cultura. Estos valores prevalecen aún en la sociedad semita que hoy día se identifica erróneamente con el mundo árabe que a su vez es considerado sinónimo del Islam.

Este legado histórico-cultural semita abarca muchos más pueblos, culturas y lenguas, diseminados por un vasto territorio que se extiende desde Egipto hasta la zona caucásica, es decir el Oriente Próximo.

En cada una de estas culturas aparecen noticias, en ocasiones fragmentarias y en otras bastante prolijas acerca de la mujer. En la antigüedad, las culturas o pueblos que nos proporcionan datos referentes a la mujer van desde Sumer, Hatti, Asiria, Babilonia, Levante, hasta Egipto. En esta fase se insiste en la defensa, los derechos y los deberes de la mujer partiendo siempre de la prevalencia de los derechos del colectivo. Por otra parte, la documentación que ha llegado hasta nosotros resalta pues todo lo relativo a lo que hoy día consideramos aspectos morales así como la situación de la mujer, declarada en muchas ocasiones un ser inferior al hombre. Esta inferioridad puede ser observada sobretodo en el campo de acción, en el cual se mueve la mujer.

Esta mentalidad se desarrollará fundamentalmente a dos niveles, el de la protección y a su vez el de su misión, que no debe entenderse en el contexto actual, partiendo de unos presupuestos nacidos y desarrollados en un territorio, donde han acaecido una serie de hechos, que nos han llevado a hablar de igualdad y libertad del ser humano 
prescindiendo de su sexo. Estos aspectos aún no han sido asimilados por la sociedad oriental semita.

Las leyes civiles antiguas que se han conservado tratan de proteger y a su vez obligar a la mujer a una serie de tradiciones que se convierten en norma oficial. Los códigos civiles tratan de regular con especial énfasis los aspectos matrimoniales, donde se insiste en la superioridad del hombre, que adquiere ciertos derechos sobre la mujer que la convierten en un sujeto que hoy día llamaríamos objeto. De hecho, muchas leyes antiguas tienen que ver con el matrimonio y la herencia como consecuencia del primer punto.

Dicho esto, se podría mencionar una realidad que los etnógrafos han denominado: matriarcado, que debe ser entendido no en sentido de que la autoridad, la ejerciera la mujer sino que se trataba de la determinación del parentesco por parte materna. Por tanto el neonato pertenecía a la familia o al clan materno. Sus derechos a la herencia, se ejercen en el seno del grupo social materno. En este sentido la mujer ejerce un derecho privilegiado pero también tiene sus inconvenientes si consideramos el caso en su conjunto. En el fondo se trataba de un fenómeno social marcado por la conservación de los bienes y la continuidad de la propia sangre en unas circunstancias condicionadas por la lucha continua entre los grupos familiares o clanes que tenían que vivir en estado de alerta continua para evitar posibles y frecuentes incursiones. En este sentido la mujer aseguraba de una forma más segura que el hombre la continuidad, ya que el papel primordial del hombre consistía en la defensa del territorio y salud de los miembros que integraba el grupo.

Esta situación irá fijando los cometidos tanto de la mujer como del hombre. Estas funciones marcarán los límites, los derechos y las obligaciones de ambos seres humanos. En esta determinación, prevalecen determinados aspectos que hacen que la mujer vaya adquiriendo un protagonismo mucho más limitado que el del hombre. Estos límites llevarán a la mujer a una situación marcada por la sumisión durante la época que se conoce como patriarcal. En no pocas ocasiones es considerada propiedad del marido. Estos aspectos aún se conservan en la cultura semita, sobre todo de tradición islámica, donde aún es considerada, sobre todo en la vida rural, como parte de las pertenencias del marido.

Ahora surge la gran pregunta: ¿Cómo ve la mujer semita su situación en esta dinámica de ser considerada perteneciente a alguien? ¿Lo sufre, lo rechaza o se ha acomodado a ello de forma pasiva y con resignación? En este caso, solo se podrá hacer unas observaciones que no pueden ir más allá de las generalidades porque tenemos constancia de casos particulares en los que la mujer semita ha roto con los esquemas preestablecidos, actuando de una forma extraordinaria y contribuyendo mejor que cualquier hombre en determinadas circunstancias al éxito de determinados objetivos 
propuestos. Por tanto, a la hora de tratar el tema en cuestión, conviene profundizar en ello desde varias perspectivas y no fijarse exclusivamente en los aspectos externos. Más adelante mencionaremos casos de este tipo, donde la mujer actúa de una forma mucho más perspicaz que el hombre y utiliza métodos que neutralizan la fuerza bruta que el semita suele emplear. Casos de este género existen entre las distintas culturas semitas, empezando desde la alta Mesopotamia hasta Egipto. Los casos más conocidos son los de Shemiran, Debora, Cleopatra y muchos otros. Estas mujeres han intervenido en un momento histórico, salvando al pueblo de peligros que podrían suponer la desaparición completa del grupo. Unas empleando el valor, otras la inteligencia y la astucia.

Pero esto no quita el lastre que pesa sobre la mujer semita si comparamos su situación con la de la mujer occidental que ha ido evolucionando de forma mucho más rápida. En esta evolución han prevalecido ciertos aspectos sociales que en primer lugar proporcionan al individuo posibilidades y ocasiones para superarse y evolucionar.

La mujer semita carece de esta proyección. Principalmente porque se ve obligada a someterse a lo que la costumbre religiosa dictamina y prescribe. Por ello su evolución, entendida en términos de la mentalidad occidental no ha pasado por las revoluciones e innovaciones que han tenido lugar tras muchos siglos de lucha e insistencia en Occidente.

Una amplia mayoría de mujeres semitas está limitada por una ley que parece ser dictada por la misma divinidad y ante la cual no cabe objeción alguna. Esta ley oprime los derechos del sexo femenino. No permite a la mujer desarrollarse libremente, porque de tener ocasión para conseguirlo, iría contra los esquemas que ha fijado la tradición que es a su vez ley y norma a seguir. Esto viniendo de lo alto, según los textos, a la mujer no le cabe otra posibilidad que acatar lo dictado porque de lo contrario, el castigo divino parece ser implacable. La convicción de que los dioses intervenían directamente en todos los asuntos de la vida social era algo muy extendido en la mentalidad semita. En la cultura semita, el hecho religioso no es objeto de especulaciones sino que se acepta tal como llega. En esto, la mujer no constituye una excepción aunque en caso de poder opinar, ciertamente no estaría de acuerdo con la norma dictada.

Evidentemente, el hecho religioso no ha proporcionado solamente deberes a la mujer sino que también derechos, que salvaguardan su integridad como "sexo débil", expuesto a una situación donde prevalecen aspectos que velan por la supervivencia y continuidad del grupo. Estos factores dieron al hombre el derecho de fijar las leyes según su forma de ver la realidad social de su época. En un documento hallado en la antigua Girsu se mencionan "reformas de Uruinimagina" que datan del período Protodinástico III (2600-2300 a.c.) y que intentan regular una situación desastrosa del estado de Lagash. Entre otras cosas habla de la mujer en términos de absoluta sumisión 
al hombre. En el documento aparece la abolición del pago de impuestos relacionados con el divorcio o los esponsales. La preeminencia del hombre sobre la mujer estaba asegurada por el soberano que ordenaba que si la mujer faltara al respeto a un hombre se le rompiera la boca con una piedra o ladrillo que posteriormente debía ser exhibido a la puerta de la ciudad. El documento dice lo siguiente:

En tiempos pasados cada mujer tenía dos hombres, pero las mujeres de nuestro tiempo han sido obligadas a abandonar es costumbre perversa.

Esto no quiere decir que el hombre ha hecho lo que le ha parecido sino que también ha dotado de medidas suficientes a la mujer para que no estuviera desamparada por completo. Hay leyes que limitan y condicionan al hombre ante la vulneración de la integridad de la mujer. El ejemplo más clásico es el código de Hammurabi que establece una serie de normas que van en defensa de la mujer. Según este código, el marido no puede tomar otra esposa salvo que se tratase de un caso de esterilidad de la primera. Tampoco podrá casarse con otra si la primera esposa le proporciona una concubina esclava que le pueda dar descendencia. Sin embargo, esta monogamia relativa en la tradición asiria y babilónica no parece ser practicada en otras culturas colindantes. El A.T. nos proporciona ejemplos en los que se habla de bigamia y poligamia en la época patriarcal. Jacob se casa con las dos hermanas Lía y Raquel, quienes a su vez le dan sus esclavas para ampliar su descendencia. Esaú contrae matrimonio con tres mujeres, dándoles el mismo rango. En épocas posteriores, tanto en Mesopotamia como en Palestina las leyes que parecían más severas, dejaron de practicarse como tales y se fueron encaminando hacia un laxismo cada vez más generalizado. En Israel, sobre todo durante la época de los jueces y bajo la monarquía, la poligamia parece ser un hecho y la bigamia un derecho. Los motivos que llevaban a casarse con más de una mujer pueden ser, en primer lugar, el ansia del hombre de tener una gran familia y por tanto no se trata de un aparente frenesí sexual del hombre. Entre otros motivos podría ponerse de relieve el de tener una sirvienta en casa. Pero como ya se ha resaltado, la razón principal para casarse por segunda vez era la prole. Solo en caso de esterilidad o de no tener un varón, el marido optaba por casarse otra vez.

El derecho asirio más tardío, parece determinar un lugar para la esposa y la concubina esclava. El código de Hammurabi se pronuncia al respecto teniendo en cuenta los problemas emocionales y sociales que podían surgir, sobre todo cuando el padre tenía hijos tanto con la esposa como con la esclava. La norma al respecto dice lo siguiente:

Si la esposa de un hombre le da hijos y su esclava también le da hijos, y si el padre reconoce en vida como hijos suyos a los hijos que le hubiera dado su esclava, y los pone en pie de igualdad con los hijos de su esposa, entonces, cuando muera el 
padre, los hijos de la esposa y los hijos de la esclava se repartirán a partes iguales la propiedad de la casa del padre; el heredero, el hijo de la esposa, sea el primero en escoger su parte. Sin embargo, si el padre no reconoce en vida como hijos suyos a los hijos que le hubiera dado la esclava, entonces, cuando el padre muera, los hijos de la esclava no se repartirán a partes iguales la propiedad de la casa del padre; la esclava y sus hijos quedarán libres, y los hijos de la esposa no reclamarán a los hijos de la esclava como esclavos suyos.

El mismo compendio legal proporciona valiosa información acerca de las mujeres fuera del contexto de la vida familiar. Las leyes hacen especial referencia a las mujeres dedicadas a las tareas de culto. Parece ser que algunas podían casarse, pero sin el derecho de poder procrear y por tanto el marido podría tener hijos con las esclavas que ellas mismas le proporcionaban. Generalmente se trataba de hijas de familias de alto rango social o incluso princesas de los estados vecinos. Su categoría social se conoce bajo el término de naditu y eran dedicadas al dios Asmas de Sipppar en calidad de prometidas. Éstas llevaban su dote consigo, al templo, y es posible que al entrar en él cambiaran de nombre. Vivían con sus servidores en una casa dentro del recinto del templo. Su principal obligación consistía en la oración por la prosperidad de su familia. Pero también intervenían en los negocios, utilizando agentes externos. De esta forma, cuando morían, la dote, enriquecida por medio de los negocios emprendidos, era devuelta a la familia de la hija entregada al templo. Aun estando entregada al templo la doncella mantenía una estrecha relación y lazos afectivos con sus familiares. En definitiva, de las normas propuestas y costumbres conservadas en la literatura asiria y babilónica se puede sostener que la mujer desempeñaba un papel importante en la sociedad. Por otra parte la elevada cantidad de leyes que hacen referencia a las distintas categorías de las mujeres, demuestra una evolución que salvaguarda los derechos y protege a la mujer en un ambiente predominantemente machista.

Semejante situación puede ser apreciada en las culturas colindantes con el vasto territorio asirio-babilónico. Entre ellos recordamos a modo de ejemplo Egipto y Hatti, donde la mujer desempeña un importante papel en la sociedad antigua. También en estas sociedades se establecen las normas para garantizar la integridad de la mujer conforme a los esquemas de una sociedad en continuo estado de evolución. En la sociedad egipcia, la mujer fue adquiriendo unos derechos que poco a poco fueron dándole una categoría social según la función que desempeñaban.

\section{MUJERES QUE HAN HECHO HISTORIA}

De lo dicho hasta el momento se podría concluir que la mujer estaba al amparo del hombre, sin iniciativa propia ni posibilidad de intervenir en la historia. Sin embargo, existen casos en los que no solo han intervenido sino también han actuado y conseguido resultados que pueden sorprender a la sociedad actual por su manera de 
actuar y razonar en determinadas circunstancias. Por tanto no solo se habla de mujer desempeñando el papel de deidad femenina o en el servicio cultual como sacerdotisas en las culturas semitas sino también tenemos constancia de la presencia de mujeres guerreras o reinas que con su modo de actuar han salvado al pueblo en situaciones de gran peligro, demostrando inteligencia, astucia y sobre todo un valor que solía atribuirse al hombre. Estas mujeres, por su actuación se convirtieron en personajes legendarios. La literatura creada en torno a su persona, ha servido a las generaciones posteriores de mujeres, sobre todo europeas para seguir luchando, y así consiguiendo los objetivos propuestos que hoy día se definen en términos de igualdad de oportunidades en las distintas esferas de la sociedad occidental. Por desgracia, no ha logrado los mismos y deseados efectos en la mujer semita. Las razones de esta actitud ya han sido tratadas anteriormente.

Las figuras de las mujeres guerreras aparecen a lo largo de la historia de los pueblos que hemos mencionado. Si tuviéramos que destacar alguna en particular, para los nororientales, sería la figura de Shammuramat, personaje histórico en el que se basa la figura legendaria de Semíramis. Esta mujer fue madre reina de Adad-nirari III (810783 a.C.) y siendo Adad-nirari menor de edad, su madre actuó como regente hasta que alcanzó la edad suficiente para ocupar el trono. El papel que desempeñó esta mujer durante los cinco largos años al frente de la corona asiria, la hizo ocupar una posición insólita, tanto que es nombrada junto con Adad-nirari III, su hijo, en una estela e incluida en una ofrenda realizada por un gobernador asirio. Los motivos de esa preeminencia podrían ser el papel fundamental que desempeñó en la defensa de la estabilidad dinástica tras la muerte de su marido, logrando la sucesión de su hijo al trono, sin los habituales incidentes graves que solían producirse en esos casos.

La literatura posterior la convierte en una heroína en la batalla, en una reina que gobierna mejor que un hombre y en una mujer que ama a su pueblo.

En un artículo del año 1853 se describe la figura de la legendaria reina asiria de una forma bastante pintoresca:

(Aquella mujer no se distinguía solo por su belleza tenía ingenio; y al seguir a su esposo, se acomodó un traje guerrero de su invención, que aumentaba sus atractivos; y al presentarse de tal modo ataviada en el campamento, todos la saludaron con admiración y aplauso.

Satisfecha en este punto su vanidad, quería lo estuviese también en alardes de valor; y en el sitio de Bactra, púsose al frente de un cuerpo de valientes asirios, y mientras los bactrianos defendían la ciudad por el sitio mas débil que era el atacado, Semíramis se dirige al lado mas difícil, escala la muralla, y lleva en pos de sí el terror y la confusión a los sitiados, que se rinden. 
Cuéntase entre sus hechos notables, que hallándose un día en su palacio peinándose, la avisaron que el pueblo se había sublevado: sin acabar de peinarse, sale a la plaza, penetra por entre la muchedumbre amotinada, y su sola presencia sosiega los ánimos y calma el tumulto. Concluido todo, se volvió tranquila a concluir su adorno. En su honor, y para recuerdo de este hecho, se erigió una estatua que la representa, con la mitad del cabello trenzado y la mitad suelto).

Evidentemente, la imagen de Shammuramat (Shammiran) o Semiramis está bastante lejos de la descripción que acabamos de oír. Esta mujer actuó como reina, dirigió como gobernanta y durante todo el tiempo que estuvo reinando mostró valor suficiente para que un ejército como era el de los asirios se sometiera y acatara sus órdenes.

\section{LA MUJER SEMITA ACTUAL}

Hasta el momento hemos tratado de ilustrar la información acerca de la imagen de la mujer según los datos que reflejan su pasado. Los datos que nos llegan, proporcionan detalles suficientes para poder afirmar que la mujer semita, por un lado se siente protegida y por el otro sometida al hombre. Este esquema nos ayuda entender su situación desde la perspectiva de una sociedad en pleno proceso de evolución en distintos frentes. Por tanto, nos encontramos en una fase histórico-social, marcada por circunstancias de continua lucha por sobrevivir en un ambiente hostil. Por ello, se subrayan determinados aspectos que condicionan factores tales como el desarrollo de la mujer en varios frentes. Al principio parece ser que no hay tiempo suficiente para una evolución que llevara a la mujer a tener las mismas posibilidades que el hombre. Los casos que hemos mencionado son excepciones y hechos aislados, donde la mujer aparece con funciones que habitualmente son desempeñados por hombres. Llegados a este punto nos preguntamos si la mujer semita actual ha progresado con el andar del tiempo. Con esta pregunta entramos en un terreno bastante resbaladizo porque los datos deben ser analizados desde una perspectiva compleja y teniendo en cuenta el amplio abanico de culturas y tradiciones ubicadas en Oriente Próximo.

Gran parte de las mujeres semitas en la actualidad pertenecen a tres grandes grupos socio-culturales condicionados por el fenómeno religioso que las condiciona y las limita en sus actuaciones a todos los niveles de vida, tanto privada como pública. La población semita, está dividida en tres grandes grupos monoteístas; los judíos, los cristianos y los musulmanes. Cada uno des estos grupos o pueblos trata a la mujer de forma distinta, teniendo en cuenta las características propias de las tres sociedades que también comparten muchos elementos socioculturales a un nivel generalizado.

Anteriormente he subrayado que la sociedad oriental no ha pasado por la evolución centenaria occidental que ha cambiado la visión de la sociedad en su conjunto. Muchos elementos nuevos han contribuido a esta apertura que nos permite hablar desde el 
punto de vista legal de igualdad de ambos sexos en términos reales. La mujer semita carece en su mayoría de los derechos o condiciones que le permiten desarrollarse como individuo. El israelita en su mayoría brinda la ocasión a un desarrollo de la mujer en los distintos niveles y se parece más bien al tipo de la mujer europea. La mujer semita cristiana, al principio parecía haber seguido un nuevo camino que le proporcionaba las mismas oportunidades que al hombre. En efecto, durante los primeros siglos de la época cristiana, la comunidad no hacía diferencia entre ambos sexos, pero a medida que se avanzaba en el tiempo fue perdiendo aquellos derechos que Cristo había propuesto para la nueva religión. De hecho, en el seno de la comunidad cristiana, la mujer parece haber pretendido desempeñar funciones, en un principio atribuidas al hombre. Esta situación debe haber provocado posibles dificultades en el seno de la antigua comunidad cristiana. Al principio, las mujeres cumplían con el mismo deber de predicar y evangelizar que el hombre. Esta novedad debe haber provocado cierto rechazo en más de una comunidad cristiana. Eco de esta circunstancia se percibe ya en las constituciones apostólicas que establecen que no se permite a las mujeres que enseñen en la iglesia dando como razón principal que Cristo, cuando compuso la lista de los elegidos no incluyó a ninguna mujer entre los doce para predicar el Evangelio, como tampoco estuvieron en la cena eucarística.... Sin embargo, en alguna zona del territorio actualmente llamado Oriente Próximo, existían comunidades cristianas que colocaban a la mujer en funciones diaconales junto al obispo y detrás del velo que separaba el altar de la nave principal durante la celebración eucarística. El diácono ejerce durante el acto litúrgico un papel muy importante. Se convierte en puente entre el pueblo y el ministro que en ese momento representa la figura de Cristo. De hecho, en la iglesia oriental de tradición lingüística aramea, la mujer tiene un lugar privilegiado durante la celebración eucarística. La arquitectura litúrgica la sitúa cerca del bema, el estrado, desde donde se celebra la primera parte de la liturgia, porque tiene más derecho que el hombre a escuchar la proclamación de la Palabra. Ello se debe al hecho de que la mujer fue la primera, a quien Cristo Resucitado se dirigió. Por otra parte, el tema del velo que la mujer solía llevar puesto durante el acto litúrgico, tiene mucho que ver con el acontecimiento que hemos mencionado. La razón principal del velo en el acto litúrgico refleja el privilegio que la mujer adquiere, por ser la primera en recibir la palabra y la luz del resucitado. De ahí que el hombre no es digno de contemplar la cara que recibió esa luz del rostro de Cristo. Por tanto, poco tiene que ver con las amonestaciones paulinas que parecen subrayar más aspectos púdicos y morales que realidades que confieren a la mujer unos privilegios que por desgracia se fueron extinguiendo por completo.

A partir de los siglos quinto y sexto y con la llegada del Islam, la mujer ha ido perdiendo terreno en este sentido. Por ello, la evolución de la mujer semita actual es muy lenta por no decir que ha quedado estancada. Es cierto que tiene más posibilidades 
ahora que antiguamente, pero no nos confundamos, con la aparente situación donde se suele decir que la mujer tiene las mismas oportunidades que en occidente. Una cosa es la teoría y otra la práctica.

El tema del velo se ha convertido en un asunto de política social en aquellos estados, donde existe una numerosa comunidad musulmana. Actualmente, el velo parece simbolizar un estilo de vida, con el que el Islam se identifica. Las veces que el Corán hace referencia al velo, en muy pocas ocasiones es utilizado en referencia a la mujer:

Cuando pidáis un objeto a sus mujeres (las del profeta), pedídselo desde detrás de una cortina. Esto es más puro para vuestros corazones y para sus corazones.

Este versículo resalta la protección de la esfera privada de las mujeres del profeta. Por lo visto su casa estaba dividida en dos partes una privada y otra publica, separadas por una cortina. Más tarde el hiyab que era exclusivamente de las mujeres de Muhammad, se convirtió en regla para todas las mujeres. En la sura 33,59 se menciona otro término Yilbab en el siguiente contexto:

“¡Profeta! Di a tus esposas, a tus hijas, a las mujeres creyentes, que se ciñan los velos (Yilbab)(cuando vayan al escusado = cuando salgan a hacer sus necesidades). Ese es el modo más sencillo de que sean reconocidas (como mujeres honestas) y no sean molestadas".

En realidad, el Corán no establece obligación religiosa general alguna acerca de la vestimenta femenina, sino sólo pautas de decencia social, en las que, por contraste con el atuendo de las beduinas y las esclavas, se recomienda como más apropiado la indumentaria urbana. El gran problema consiste en la interpretación de forma mucho más restrictiva de los pasajes coránicos mencionados. Las dos escuelas jurídicas mayoritarias sunnies y chiíes, interpretan los versículos coránicos citados, viendo en ellos una prescripción religiosa que obliga a las mujeres a llevar el velo. Los círculos más progresistas de la sociedad musulmana actual entienden las azoras como si fueran instrucciones impuestas por la cultura y la época. Por ello este sector de la sociedad musulmana considera que el uso de esta prenda no es obligatorio para todas las mujeres musulmanas. En algunos estados islámicos, como es el caso de Irán o Afganistán...se aplica como un deber generalizado y la mujer no tiene ninguna opción de vulnerarlo. En mi opinión, la razón principal de esta insistencia consiste en el rechazo de todo lo que procede del mundo occidental. Los fundamentalistas quieren recuperar la vestimenta islámica para las mujeres, no para recuperar la costumbre indumentaria islámica tradicional sino más bien para evitar que la mujer musulmana se vista al igual que una europea.

En muchos estados islámicos la mujer está totalmente desamparada en cuanto a las posibilidades de desarrollo. Hay grupos que impiden que la mujer pueda tomar sus 
propias iniciativas y decisiones en los distintos ámbitos de la vida. La ley coránica no concede a la mujer los mismos derechos que al hombre. Por tanto, la evolución de la mujer entendida en términos actuales, es prácticamente inexistente.

Podríamos formular como interrogante principal el siguiente. ¿Cómo se siente y como actúa la mujer musulmana semita en la sociedad del siglo XXI? Aquí cabe distinguir dos grupos femeninos en la sociedad islámica. La mujer en el ámbito rural y en el urbano. La mujer que vive en el ámbito urbano, lógicamente tiene muchas más posibilidades para acceder a una educación y posible evolución, sin embargo la mujer semita rural vive bajo los mismos esquemas milenarios y costumbres tradicionales que no le permiten tener las mismas posibilidades que las mujeres que viven en grandes ciudades. Por tanto si uno nace, crece y muere con los mismos esquemas y en las mismas condiciones ancestrales no notará o sufrirá las desigualdades de las que es víctima. Este grupo de mujeres llega a ver estas circunstancias como si fueran normales y las únicas válidas y dignas de seguirse. A nadie le gusta ser repudiado pero si la norma lo establece, no cabe otra opción que acatarla. Si la tradición establece que la mujer tiene que llevar el velo o el burca, aunque dañe a la vista, pues hay que llevarlo. Si la ley establece que el marido puede casarse con más de una mujer, es la divinidad quien lo establece y por tanto el hombre hace uso de su legítimo derecho...

La resignación de la mujer musulmana es una realidad y en otras ocasiones, ella misma cree que es lo que debe ser. Los conceptos de evolución son totalmente opuestos, son visiones absolutamente contrapuestas. La mujer con esta mentalidad no se contempla y ni entiende a sí misma sin su entorno, que vela exclusivamente por los intereses del colectivo o del grupo. El individuo no existe en este sentido, mucho menos la mujer, cuya misión principal consiste en ampliar el número de la prole que debe crecer y multiplicarse. Este es el gran drama de la mujer musulmana que en cierta medida, consciente de ello, no se atreve a pronunciarse públicamente porque las consecuencias podrían resultar terribles. Esto por una parte, y el respeto por la otra impiden que la mujer tenga la posibilidad de abrirse camino en una sociedad que en su gran mayoría controla el "macho" que goza de ciertos derechos inaccesibles a la mujer. La revolución llegará también a la mujer semita en este sentido pero tardará mucho tiempo en alcanzar sus objetivos e ilusiones, oprimidas por el momento, pero con la esperanza de que los logre para las futuras generaciones. Es cuando empezará la gran revolución y se producirán grandes cambios para la mayor parte de las mujeres semitas.

\section{REFERENCIAS BIBLIOGRÁFICAS}

Cooper, J. S., Sumerian and Akkadian Royal inscriptions, vol. I: Pre-Sargonic Inscriptions, Winona Lake, 1986, 9.3. 
Dalley, S., Mari and Karana: two Old Babylonian Cities, London, 1984, p. 105.

De Vaux, R., Instituciones del Antiguo Testamento, Barcelona, 1985, pp. 49-93.

Harris, R., 'Independent women in ancient Mesopotamia?', Leski (1989) 145-156.

Koch, Y. M. (ed.), La mujer judía, Córdoba, 2007.

Kuhrt, A., El Oriente Próximo en la antigüedad, I, Barcelona, 2000, p. 57.

Perala, A., 'Semiramis', El correo de la Moda 22 (1853). 